\title{
Variation in quality of preventive care for well adults in Indigenous community health centres in Australia
}

Ross S Bailie ${ }^{1}$, Damin Si ${ }^{1,2^{*}}$, Christine M Connors ${ }^{3}$, Ru Kwedza $^{4}$, Lynette O'Donoghue ${ }^{1,3}$, Catherine Kennedy ${ }^{5}$, Rhonda Cox ${ }^{6}$, Helen Liddle1, Jenny Hains', Michelle C Dowden', Hugh P Burke ${ }^{5}$, Alex Brown?

Tarun Weeramanthri ${ }^{8}$ and Sandra Thompson ${ }^{6,9}$

\begin{abstract}
Background: Early onset and high prevalence of chronic disease among Indigenous Australians call for action on prevention. However, there is deficiency of information on the extent to which preventive services are delivered in Indigenous communities. This study examined the variation in quality of preventive care for well adults attending Indigenous community health centres in Australia.

Methods: During 2005-2009, clinical audits were conducted on a random sample (stratified by age and sex) of records of adults with no known chronic disease in 62 Indigenous community health centres in four Australian States/Territories (sample size 1839). Main outcome measures: i) adherence to delivery of guideline-scheduled services within the previous 24 months, including basic measurements, laboratory investigations, oral health checks, and brief intervention on lifestyle modification; and ii) follow-up of abnormal findings.

Results: Overall delivery of guideline-scheduled preventive services varied widely between health centres (range 574\%). Documentation of abnormal blood pressure reading ([greater than or equal to]140/90 $\mathrm{mmHg}$ ), proteinuria and abnormal blood glucose ([greater than or equal to] $5.5 \mathrm{mmol} / \mathrm{L}$ ) was found to range between 0 and $>90 \%$ at the health centre level. A similarly wide range was found between health centres for documented follow up check/test or management plan for people documented to have an abnormal clinical finding. Health centre level characteristics explained $13-47 \%$ of variation in documented preventive care, and the remaining variation was explained by client level characteristics.

Conclusions: There is substantial room to improve preventive care for well adults in Indigenous primary care settings. Understanding of health centre and client level factors affecting variation in the care should assist clinicians, managers and policy makers to develop strategies to improve quality of preventive care in Indigenous communities.
\end{abstract}

\section{Background}

As part of the response to high levels of chronic disease among Indigenous Australians[1], there has been increasing emphasis in recent years on delivery of preventive services in Indigenous primary health care services. This includes development and distribution of evidence-based, Indigenous population specific preventive care guidelines

\footnotetext{
* Correspondence: damin.si@menzies.edu.au

'Menzies School of Health Research, Charles Darwin University, Darwin NT, Australia

Full list of author information is available at the end of the article
}

[2], introduction of Medicare reimbursed biennial health checks for Indigenous adults aged 15 years or over [3], and the newly released National Preventative Health Strategy which specifies targets and actions for multifaceted preventive care to "close the gap" in life expectancy between Indigenous and other Australians [4].

Previous research provides limited information on delivery of preventive care in Indigenous primary care settings. Studies conducted in the Northern Territory (NT) have documented substantial deficiencies in delivery of preventive care to Indigenous adults in rural and

\section{Biomed Central}


remote communities: on average only $40-50 \%$ of preventive services were delivered in line with the best practice guidelines [5,6]. Studies in Indigenous communities in Queensland (QLD) $[7,8]$ have not included data on the proportion of community members who received health checks. A national study using Medicare data showed that 3\% of Indigenous Australians aged 55 years or over attending GPs had documented use of specific Medicare items for health checks [9]. However, the study appeared to underestimate the uptake of preventive health checks as many Indigenous primary care services do not use Medicare items when delivering services to clients. Thus there is substantial potential to improve the quality of information on the delivery of preventive services to Indigenous people for the purpose of informing implementation of the National Preventative Health Strategy.

The Audit and Best-practice for Chronic Disease Extension (ABCDE) project is a national quality improvement initiative which aims to improve quality of care in a range of priority aspects of Indigenous primary health care, including chronic disease care, preventive care, and maternal and child health care [10]. During the past five years over 60 Indigenous community health centres from four States/Territories (NT, Far West New South Wales (NSW), Western Australia (WA) and North QLD) have formally participated in this project. The ABCDE data provide a unique opportunity to improve understanding of delivery of preventive care in Indigenous primary health care settings, and, importantly, to develop and implement strategies for improvement. This paper reports baseline data on delivery of preventive care in Indigenous community health centres participating in $A B C D E$ with a focus on variation in quality of care between services and across different participating regions, and identifies the various factors associated with these variations, both at the health centre level and at the individual level.

\section{Methods}

Participation by health centres was from five regions where we had established project hub coordinators (Figure 1 and Table 1). On a voluntary basis, health centre managers or staff made a request for their centre to join the project after receiving information through invitation letters, word of mouth or meeting presentations. Sixty six (66) health centres formally participated in the $\mathrm{ABCDE}$ project. Four (4) of these health centres did not have at least part time access to a GP and were excluded from the analysis for this paper.

Baseline audits of preventive care were completed during 2005-2009. Audits covered both paper-based and electronic clinical records. The records of health centre clients who met all of the following criteria were eligible

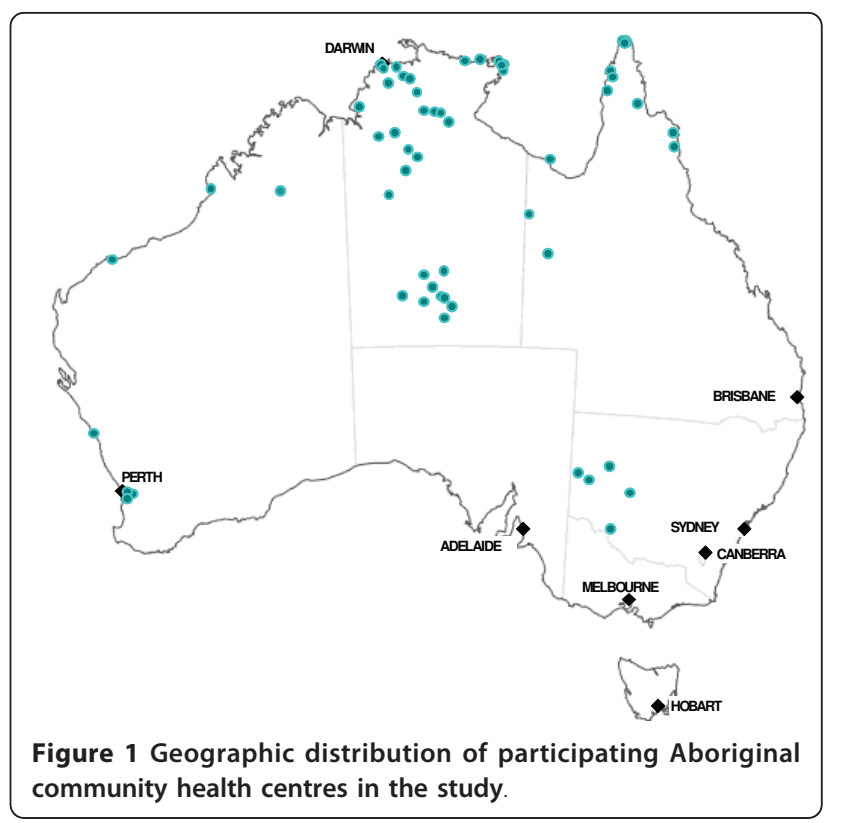

for inclusion: 1) aged between 15 and 54 years; 2) resident in the community for at least 6 of the last 12 months; 3) not having a diagnosis of diabetes, hypertension, ischaemic heart disease, rheumatic heart disease, renal disease or other major chronic illness; and 4) not pregnant or post partum at the time of the audit. Eligibility was verified by checking an up-to-date population list of health centre clients with assistance from health centre staff who knew the community and health centre well. A sample of 30 records, stratified by sex and age groups (15-24; 25-39; and 40-54 years), was selected randomly from records of eligible clients in each health centre. Thus, each random sample comprised 5 males and 5 females in each of the age groups. In communities where there were fewer than 5 people in a sex- and agespecific group, all eligible people in that group were included.

The audit measured 16 selected service items (see Table 2) which the preventive health care guidelines recommend for delivery every year or every two years for all Indigenous well adults aged $15-54$ years $[2,11]$. A summary of detailed guideline recommendations in relation to the 16 service items is presented in Table 3 . We adopted a minimum approach to assess whether these services were delivered on a two-yearly basis. A service was assessed as delivered if there was a clear record of delivery of the service at least once within the previous 24 months. The overall adherence to delivery of scheduled services for each adult was calculated by dividing the sum of services delivered by 16 (for females) or 15 (for males - pap smear excluded), and expressing this as a percentage. For example, if there were 6 services assessed as delivered for a male client, the overall 
Table 1 Characteristics of participating health centres and adults

\begin{tabular}{|c|c|c|c|c|c|c|}
\hline Characteristic & $\begin{array}{l}\text { NT Top } \\
\text { End }\end{array}$ & $\begin{array}{l}\text { NT Central } \\
\text { Australia }\end{array}$ & $\begin{array}{l}\text { Far West } \\
\text { NSW }\end{array}$ & WA & $\begin{array}{l}\text { North } \\
\text { QLD }\end{array}$ & Total \\
\hline Community health centres & 24 & 9 & 5 & 7 & 17 & 62 \\
\hline \multicolumn{7}{|l|}{ Locations } \\
\hline City & $1(4 \%)$ & $1(11 \%)$ & $0(0 \%)$ & $3(43 \%)$ & $0(0 \%)$ & $5(8 \%)$ \\
\hline Regional town & $6(25 \%)$ & $0(0 \%)$ & $4(80 \%)$ & $2(28 \%)$ & $0(0 \%)$ & $12(19 \%)$ \\
\hline Remote community & $17(71 \%)$ & $8(89 \%)$ & $1(20 \%)$ & $2(29 \%)$ & $\begin{array}{c}17 \\
(100 \%)\end{array}$ & $45(73 \%)$ \\
\hline \multicolumn{7}{|l|}{ Health service governance } \\
\hline Government funded/operated & $10(42 \%)$ & $5(56 \%)$ & $0(0 \%)$ & $1(14 \%)$ & $\begin{array}{c}17 \\
(100 \%)\end{array}$ & $33(53 \%)$ \\
\hline $\begin{array}{l}\text { Managed by local or regional Indigenous } \\
\text { committee or board }\end{array}$ & $14(58 \%)$ & $4(44 \%)$ & $5(100 \%)$ & $6(86 \%)$ & $0(0 \%)$ & $29(47 \%)$ \\
\hline \multicolumn{7}{|l|}{ General practice accreditation status } \\
\hline Currently accredited & $9(38 \%)$ & $3(33 \%)$ & $1(20 \%)$ & $2(29 \%)$ & $5(29 \%)$ & 19 (31\%) \\
\hline Not accredited & $15(62 \%)$ & $6(67 \%)$ & $4(80 \%)$ & $5(71 \%)$ & $12(71 \%)$ & $43(69 \%)$ \\
\hline \multicolumn{7}{|l|}{ Sizes of populations served } \\
\hline$\leq 500$ & $9(37 \%)$ & $4(44 \%)$ & $1(20 \%)$ & $0(0 \%)$ & $6(35 \%)$ & $20(32 \%)$ \\
\hline $501-999$ & $6(25 \%)$ & $4(45 \%)$ & $3(60 \%)$ & $0(0 \%)$ & $4(24 \%)$ & $17(28 \%)$ \\
\hline$\geq 1000$ & $9(38 \%)$ & $1(11 \%)$ & $1(20 \%)$ & $7(100 \%)$ & $7(41 \%)$ & $25(40 \%)$ \\
\hline Participating adults & 708 & 267 & 150 & 210 & 504 & 1839 \\
\hline Mean age (years) & 32 & 31 & 33 & 32 & 33 & 32 \\
\hline Males & $49 \%$ & $49 \%$ & $50 \%$ & $50 \%$ & $50 \%$ & $49 \%$ \\
\hline \multicolumn{7}{|l|}{ Indigenous status } \\
\hline Indigenous & $94 \%$ & $95 \%$ & $43 \%$ & $87 \%$ & $91 \%$ & $89 \% *$ \\
\hline Non-Indigenous & $4 \%$ & $1 \%$ & $50 \%$ & $10 \%$ & $1 \%$ & $7 \% *$ \\
\hline Not stated & $2 \%$ & $4 \%$ & $7 \%$ & $3 \%$ & $8 \%$ & $4 \%$ \\
\hline $\begin{array}{l}\text { Attended health centre within the past } 24 \text { months (range between } \\
\text { health centres) }\end{array}$ & $\begin{array}{c}93 \% \\
(50 \%- \\
100 \%)\end{array}$ & $\begin{array}{c}97 \% \\
(90-100 \%)\end{array}$ & $\begin{array}{c}75 \% \\
(30 \%-93 \%)\end{array}$ & $\begin{array}{c}99 \% \\
(93 \%- \\
100 \%)\end{array}$ & $\begin{array}{c}90 \% \\
(53 \%- \\
100 \%)\end{array}$ & $\begin{array}{l}92 \% *{ }^{*} \\
(30 \%- \\
100 \%)\end{array}$ \\
\hline \multicolumn{7}{|l|}{ Reasons for last attendance } \\
\hline Well person's check & $13 \%$ & $10 \%$ & $4 \%$ & $3 \%$ & $4 \%$ & $8 \% *$ \\
\hline Acute care & $58 \%$ & $56 \%$ & $42 \%$ & $59 \%$ & $53 \%$ & $55 \% *$ \\
\hline Immunisation & $2 \%$ & $2 \%$ & $4 \%$ & $1 \%$ & $12 \%$ & $5 \%$ \\
\hline Sexual health & $7 \%$ & $9 \%$ & $4 \%$ & $3 \%$ & $6 \%$ & $6 \%$ \\
\hline Mental health & $1 \%$ & $1 \%$ & $5 \%$ & $0 \%$ & $2 \%$ & $1 \%$ \\
\hline others & $19 \%$ & $22 \%$ & $41 \%$ & $34 \%$ & $23 \%$ & $25 \% *$ \\
\hline \multicolumn{7}{|l|}{ Key responsible health providers during the last attendance } \\
\hline Nurses & $48 \%$ & $64 \%$ & $39 \%$ & $13 \%$ & $55 \%$ & $47 \% *$ \\
\hline Aboriginal health workers & $26 \%$ & $14 \%$ & $7 \%$ & $13 \%$ & $15 \%$ & $18 \% \%^{*}$ \\
\hline Doctors & $14 \%$ & $12 \%$ & $40 \%$ & $62 \%$ & $9 \%$ & $20 \% *$ \\
\hline Specialists & $1 \%$ & $1 \%$ & $4 \%$ & $2 \%$ & $2 \%$ & $1 \%$ \\
\hline Others & $11 \%$ & $9 \%$ & $10 \%$ & $10 \%$ & $19 \%$ & $14 \%$ \\
\hline Documented as a smoker & $30 \%$ & $23 \%$ & $27 \%$ & $28 \%$ & $29 \%$ & $28 \%$ \\
\hline Documented alcohol misuse & $15 \%$ & $15 \%$ & $27 \%$ & $12 \%$ & $40 \%$ & $23 \% *$ \\
\hline Documented organic complications of alcohol misuse & $5 \%$ & $7 \%$ & $3 \%$ & $4 \%$ & $13 \%$ & $7 \% *$ \\
\hline
\end{tabular}

* $\mathrm{P}<0.05$ for comparison between regions.

adherence to delivery of services for the client was $40 \%$ $(6 / 15)$, interpreted as " $40 \%$ of guidelines-scheduled preventive services were delivered to the client". Health centre-level adherence was computed as the mean of individual adherence to delivery at each centre. For each individual service item, a percentage (from 0 to $100 \%$ ) was calculated at each health centre to represent "\% of patients who received the specific preventive service".

Clinical records were also audited for evidence of abnormal blood pressure readings, positive protein in urine and abnormal blood glucose readings. For any abnormality found we checked for a record of a follow- 
Table 2 Mean adherence to delivery of scheduled services for 62 community health centres within previous 24 months

\begin{tabular}{|c|c|c|c|c|c|c|}
\hline \multirow[b]{2}{*}{ Process item } & $\begin{array}{l}\text { NT } \\
\text { Top End }\end{array}$ & NT Central Australia & $\begin{array}{l}\text { Far West } \\
\text { NSW }\end{array}$ & WA & $\begin{array}{l}\text { North } \\
\text { QLD }\end{array}$ & Total \\
\hline & \multicolumn{6}{|c|}{$\begin{array}{c}\text { Mean percentages } \pm \text { SE } \\
\text { (range between health centres) }\end{array}$} \\
\hline Total No. of centres | adults & $24 \mid 708$ & $9 \mid 267$ & 5 | 150 & $7 \mid 210$ & $17 \mid 504$ & $62 \mid 1839$ \\
\hline \multicolumn{7}{|l|}{ Basic measurements } \\
\hline Weight & $\begin{array}{l}56 \% \pm 5 \% \\
(10 \%-100 \%)\end{array}$ & $\begin{array}{l}58 \% \pm 7 \% \\
(18 \%-83 \%)\end{array}$ & $\begin{array}{l}28 \% \pm 7 \% \\
(0 \%-40 \%)\end{array}$ & $\begin{array}{l}52 \% \pm 9 \% \\
(17 \%-73 \%)\end{array}$ & $\begin{array}{l}61 \% \pm 5 \% \\
(28 \%-87 \%)\end{array}$ & $\begin{array}{l}55 \% \pm 3 \% * \\
(0 \%-100 \%)\end{array}$ \\
\hline Height & $\begin{array}{l}40 \% \pm 6 \% \\
(3 \%-100 \%)\end{array}$ & $\begin{array}{l}40 \% \pm 9 \% \\
(4 \%-77 \%)\end{array}$ & $\begin{array}{l}23 \% \pm 6 \% \\
(0 \%-40 \%)\end{array}$ & $\begin{array}{l}47 \% \pm 10 \% \\
(3 \%-70 \%)\end{array}$ & $\begin{array}{l}39 \% \pm 3 \% \\
(17 \%-63 \%)\end{array}$ & $\begin{array}{l}39 \% \pm 3 \% \\
(0 \%-100 \%)\end{array}$ \\
\hline BMI & $\begin{array}{c}26 \% \pm 6 \% \\
(0 \%-87 \%)\end{array}$ & $\begin{array}{c}25 \% \pm 9 \% \\
(0 \%-77 \%)\end{array}$ & $\begin{array}{l}15 \% \pm 6 \% \\
(0 \%-33 \%)^{* *}\end{array}$ & $\begin{array}{c}27 \% \pm 8 \% \\
(3 \%-60 \%)\end{array}$ & $\begin{array}{l}15 \% \pm 4 \% \\
(0 \%-57 \%)\end{array}$ & $\begin{array}{c}22 \% \pm 3 \% \\
(0 \%-87 \%)\end{array}$ \\
\hline Waist circumference & $\begin{array}{l}26 \% \pm 5 \% \\
(0 \%-85 \%)\end{array}$ & $\begin{array}{c}28 \% \pm 8 \% \\
(0 \%-60 \%)\end{array}$ & $\begin{array}{l}15 \% \pm 6 \% \\
(0 \%-33 \%)^{* *}\end{array}$ & $\begin{array}{l}11 \% \pm 7 \% \\
(0 \%-57 \%)\end{array}$ & $\begin{array}{l}17 \% \pm 3 \% \\
(0 \%-57 \%)\end{array}$ & $\begin{array}{c}21 \% \pm 3 \% \\
(0 \%-85 \%)\end{array}$ \\
\hline Blood pressure & $\begin{array}{l}71 \% \pm 4 \% \\
(23 \%-100 \%)\end{array}$ & $\begin{array}{l}75 \% \pm 6 \% \\
(33 \%-90 \%)\end{array}$ & $\begin{array}{l}55 \% \pm 9 \% \\
(23 \%-73 \%)\end{array}$ & $\begin{array}{l}65 \% \pm 9 \% \\
(27 \%-93 \%)\end{array}$ & $\begin{array}{l}76 \% \pm 4 \% \\
(41 \%-97 \%)\end{array}$ & $\begin{array}{l}71 \% \pm 3 \% \\
(23 \%-100 \%)\end{array}$ \\
\hline \multicolumn{7}{|l|}{ Laboratory investigations } \\
\hline Urinalysis & $\begin{array}{l}43 \% \pm 5 \% \\
(8 \%-97 \%)\end{array}$ & $\begin{array}{l}46 \% \pm 8 \% \\
(11 \%-77 \%)\end{array}$ & $\begin{array}{c}13 \% \pm 5 \% \\
(7 \%-30 \%)\end{array}$ & $\begin{array}{l}34 \% \pm 10 \% \\
(13 \%-80 \%)\end{array}$ & $\begin{array}{l}42 \% \pm 4 \% \\
(21 \%-80 \%)\end{array}$ & $\begin{array}{c}40 \% \pm 3 \% * \\
(7 \%-97 \%)\end{array}$ \\
\hline Blood glucose level & $\begin{array}{l}56 \% \pm 5 \% \\
(13 \%-97 \%)\end{array}$ & $\begin{array}{l}56 \% \pm 9 \% \\
(17 \%-83 \%)\end{array}$ & $\begin{array}{l}35 \% \pm 8 \% \\
(7 \%-53 \%)^{* *}\end{array}$ & $\begin{array}{l}53 \% \pm 8 \% \\
(20 \%-77 \%)\end{array}$ & $\begin{array}{l}65 \% \pm 4 \% \\
(41 \%-90 \%)\end{array}$ & $\begin{array}{c}56 \% \pm 3 \% \\
(7 \%-97 \%)\end{array}$ \\
\hline Pap smear (women only) & $\begin{array}{c}42 \% \pm 5 \% \\
(0 \%-80 \%)\end{array}$ & $\begin{array}{c}44 \% \pm 8 \% \\
(6 \%-93 \%)\end{array}$ & $\begin{array}{c}28 \% \pm 9 \% \\
(0 \%-53 \%)\end{array}$ & $\begin{array}{c}28 \% \pm 7 \% \\
(0 \%-56 \%)\end{array}$ & $\begin{array}{l}42 \% \pm 4 \% \\
(13 \%-60 \%)\end{array}$ & $\begin{array}{c}40 \% \pm 3 \% \\
(0 \%-93 \%)\end{array}$ \\
\hline STI: PCR for gonorrhoea \& chlamydia & $\begin{array}{c}44 \% \pm 6 \% \\
(0 \%-93 \%)\end{array}$ & $\begin{array}{l}57 \% \pm 7 \% \\
(20 \%-83 \%)\end{array}$ & $\begin{array}{l}5 \% \pm 2 \% \\
(0 \%-10 \%)\end{array}$ & $\begin{array}{c}25 \% \pm 10 \% \\
(0 \%-73 \%)\end{array}$ & $\begin{array}{c}43 \% \pm 5 \% \\
(4 \%-87 \%)\end{array}$ & $\begin{array}{c}40 \% \pm 3 \% * \\
(0 \%-93 \%)\end{array}$ \\
\hline STI: Syphilis serology & $\begin{array}{c}36 \% \pm 5 \% \\
(0 \%-93 \%)\end{array}$ & $\begin{array}{l}57 \% \pm 8 \% \\
(18 \%-90 \%)\end{array}$ & $\begin{array}{l}4 \% \pm 3 \% \\
(0 \%-13 \%)\end{array}$ & $\begin{array}{c}16 \% \pm 9 \% \\
(0 \%-57 \%)\end{array}$ & $\begin{array}{c}35 \% \pm 5 \% \\
(0 \%-90 \%)\end{array}$ & $\begin{array}{c}34 \% \pm 3 \% * \\
(0 \%-93 \%)\end{array}$ \\
\hline Oral health check & $\begin{array}{c}18 \% \pm 3 \% \\
(0 \%-63 \%)\end{array}$ & $\begin{array}{c}16 \% \pm 7 \% \\
(0 \%-67 \%)\end{array}$ & $\begin{array}{c}11 \% \pm 5 \% \\
(0 \%-23 \%)\end{array}$ & $\begin{array}{c}17 \% \pm 9 \% \\
(0 \%-67 \%)\end{array}$ & $\begin{array}{c}10 \% \pm 4 \% \\
(0 \%-50 \%)\end{array}$ & $\begin{array}{c}15 \% \pm 2 \% \\
(0 \%-67 \%)\end{array}$ \\
\hline \multicolumn{7}{|l|}{ Brief intervention/advice } \\
\hline Smoking & $\begin{array}{c}21 \% \pm 4 \% \\
(0 \%-73 \%)\end{array}$ & $\begin{array}{c}35 \% \pm 8 \% \\
(3 \%-70 \%)\end{array}$ & $\begin{array}{l}20 \% \pm 6 \% \\
(10 \%-43 \%)\end{array}$ & $\begin{array}{c}17 \% \pm 6 \% \\
(3 \%-53 \%)\end{array}$ & $\begin{array}{l}39 \% \pm 4 \% \\
(10 \%-75 \%)\end{array}$ & $\begin{array}{c}28 \% \pm 3 \% * \\
(0 \%-75 \%)\end{array}$ \\
\hline Nutrition & $\begin{array}{c}17 \% \pm 5 \% \\
(0 \%-81 \%)\end{array}$ & $\begin{array}{c}36 \% \pm 8 \% \\
(7 \%-67 \%)\end{array}$ & $\begin{array}{c}13 \% \pm 4 \% \\
(3 \%-23 \%)\end{array}$ & $\begin{array}{c}12 \% \pm 6 \% \\
(0 \%-47 \%)\end{array}$ & $\begin{array}{c}34 \% \pm 4 \% \\
(8 \%-63 \%)\end{array}$ & $\begin{array}{c}24 \% \pm 3 \% * \\
(0 \%-81 \%)\end{array}$ \\
\hline Alcohol & $\begin{array}{c}20 \% \pm 4 \% \\
(0 \%-73 \%)\end{array}$ & $\begin{array}{l}37 \% \pm 7 \% \\
(13 \%-67 \%)\end{array}$ & $\begin{array}{c}18 \% \pm 6 \% \\
(7 \%-40 \%)\end{array}$ & $\begin{array}{c}17 \% \pm 6 \% \\
(3 \%-53 \%)\end{array}$ & $\begin{array}{l}41 \% \pm 4 \% \\
(17 \%-75 \%)\end{array}$ & $\begin{array}{c}28 \% \pm 3 \% * \\
(0 \%-75 \%)\end{array}$ \\
\hline Physical activity & $\begin{array}{c}17 \% \pm 5 \% \\
(0 \%-88 \%)\end{array}$ & $\begin{array}{c}36 \% \pm 8 \% \\
(0 \%-67 \%)\end{array}$ & $\begin{array}{c}11 \% \pm 4 \% \\
(3 \%-27 \%)\end{array}$ & $\begin{array}{c}14 \% \pm 7 \% \\
(3 \%-53 \%)\end{array}$ & $\begin{array}{c}31 \% \pm 4 \% \\
(0 \%-63 \%)\end{array}$ & $\begin{array}{c}23 \% \pm 3 \% * \\
(0 \%-88 \%)\end{array}$ \\
\hline Mood (emotional well-being) & $\begin{array}{l}17 \% \pm 5 \% \\
(0 \%-81 \%)\end{array}$ & $\begin{array}{c}28 \% \pm 7 \% \\
(0 \%-67 \%)\end{array}$ & $\begin{array}{l}15 \% \pm 8 \% \\
(3 \%-43 \%)^{* *}\end{array}$ & $\begin{array}{l}15 \% \pm 6 \% \\
(3 \%-47 \%)\end{array}$ & $\begin{array}{c}18 \% \pm 4 \% \\
(0 \%-50 \%)\end{array}$ & $\begin{array}{l}18 \% \pm 2 \% \\
(0 \%-81 \%)\end{array}$ \\
\hline Overall adherence & $\begin{array}{c}34 \% \pm 4 \% \\
(8 \%-74 \%)\end{array}$ & $\begin{array}{l}42 \% \pm 7 \% \\
(15 \%-71 \%)\end{array}$ & $\begin{array}{c}19 \% \pm 5 \% \\
(5 \%-32 \%)\end{array}$ & $\begin{array}{l}28 \% \pm 6 \% \\
(13 \%-59 \%)\end{array}$ & $\begin{array}{l}38 \% \pm 3 \% \\
(21 \%-65 \%)\end{array}$ & $\begin{array}{c}34 \% \pm 3 \% \\
(5 \%-74 \%)\end{array}$ \\
\hline
\end{tabular}

* $\mathrm{P}<0.05$ for comparison between regions.

** indicating statistically significant differences $(P<0.05)$ between Indigenous and non-Indigenous adults in Far West NSW, as detailed below: BMI (Indigenous $23 \%$ vs non-Indigenous $8 \%, P=0.03$ ); waist ( $22 \%$ vs $8 \%, P=0.04)$; blood glucose level ( $48 \%$ vs $25 \%, P=0.04)$; and $\operatorname{mood}(22 \%$ vs $11 \%, P=0.02)$.

Percentages are based on standard stratified samples from each health centre with equal numbers of males and females in three age bands and are not weighted for population size.

up as outlined in Table 4. A percentage was calculated for each health centre to represent "\% of adults who had appropriate follow-up of abnormal findings".

\section{Statistical analysis}

The quality of preventive care was measured in terms of adherence to delivery of scheduled services and followup of abnormal findings. Treating health centres as the unit of analysis, we compared the quality of care (based on mean percentages) between regions using linear regression models (Tables 2 and 4). Centre percentages and mean percentages are unweighted.

When treating individual clients as the unit of analysis, our data had inherent multilevel, dependency structure, as preventive care data collected at the individual client level (level 1) were clustered within health centres (level 2). Two-level random effects regression models (linear or logistic) [12] were used to 1) quantify the 
Table 3 Recommended schedule for the adult well person's health check for Indigenous people

\begin{tabular}{|c|c|c|c|}
\hline Service item & Guideline & Frequency & Starting age \\
\hline \multicolumn{4}{|l|}{ Basic measurements } \\
\hline \multirow[t]{2}{*}{ Weight } & A & Yearly & 10 years \\
\hline & B & Yearly & 15 years \\
\hline Height & B & $1^{\text {st }}$ visit & \\
\hline \multirow[t]{2}{*}{ BMI } & A & Yearly & 10 years \\
\hline & B & Yearly & 15 years \\
\hline \multirow[t]{2}{*}{ Waist circumference } & A & Yearly (6-monthly in obesity/overweight) & 18 years \\
\hline & B & Yearly & 15 years \\
\hline \multirow[t]{2}{*}{ Blood pressure } & A & Check every visit, at least yearly & 18 years or earlier \\
\hline & B & Yearly & 15 years \\
\hline \multicolumn{4}{|l|}{ Laboratory investigations } \\
\hline \multirow[t]{2}{*}{ Urinalysis } & A & Yearly & $15-18$ years \\
\hline & B & Yearly & 15 years \\
\hline \multirow[t]{2}{*}{ Blood glucose level } & A & 1-2 yearly & $15-18$ years in high prevalence \\
\hline & B & Yearly & 15 years \\
\hline Pap smear (women only) & A & 2-yearly & Women aged 18-70 \\
\hline \multirow[t]{2}{*}{ STI: PCR for gonorrhoea \& chlamydia } & A & $1-2$ yearly & $<25$ years for sexually active men and women \\
\hline & B & Yearly & 15 years \\
\hline \multirow[t]{2}{*}{ STI: Syphilis serology } & A & $1-2$ yearly & $15-30$ years \\
\hline & B & Yearly & 15 years \\
\hline Oral health check & A & Yearly & All ages \\
\hline \multicolumn{4}{|l|}{ Brief intervention/advice } \\
\hline \multirow[t]{2}{*}{ Smoking } & A & Yearly & 10 years \\
\hline & B & Yearly & 15 years \\
\hline Nutrition & B & Yearly & 15 years \\
\hline \multirow[t]{2}{*}{ Alcohol } & A & Yearly & $14-15$ years \\
\hline & B & Yearly & 15 years \\
\hline Physical activity & B & Yearly & 15 years \\
\hline Mood (emotional well-being) & A & Every visit & 15 years \\
\hline
\end{tabular}

A - National guide to a preventive health assessment in Aboriginal and Torres Strait Islander peoples [2].

B - CARPA standard treatment manual: a clinic manual for primary health care practitioners in remote and rural communities in Central and Northern Australia [11].

amount of variation attributable to health centre and individual level characteristics (Table 5); and 2) examine associations of specific factors with quality of preventive care (Tables 6 and 7), as outlined below:

1) Adherence to delivery of services and follow up of abnormal findings were treated as dependent variables in the random effects models respectively. We constructed a two-level (health centre and client levels) random intercept model with no explanatory variables (also known as an empty model) [13]. In the context of multilevel modelling, the empty model provides an estimate of the basic partition of the variability in the data between the two levels. Based on the model, an intraclass correlation coefficient (rho in Stata [12]) between two randomly drawn individuals in a given health centre was estimated. The intra-class correlation coefficient can also be interpreted as the fraction of total variability in the dependent variable that is due to health centre level characteristics. The remaining variation is attributable to client level characteristics. The term "characteristics" used here refers to measured and un-measured factors at the health centre and client levels.

2) Using two-level random effects regression models, we also tested associations of specific factors at health centre (location, health service governance, accreditation status and population size) and individual levels (age and sex) with the quality of preventive care.

We obtained approval from formally constituted Human Research Ethics Committees (HREC, including Indigenous health research committees where such arrangements were in place) in each region in which the project operated, including the NT Department of Health \& Community Services and Menzies School of Health Research HREC, the Central Australian HREC, the Western Australian Aboriginal Health Information and Ethics Committee, the Macquarie and Far West Area Health Services HREC, and the Townsville Health Service District HREC. 
Table 4 Follow-up of abnormal clinical findings at participating health centres

\begin{tabular}{|c|c|c|c|c|c|c|}
\hline \multirow[b]{2}{*}{ Abnormal findings and follow ups } & \multirow[t]{2}{*}{ NT Top End } & \multirow[t]{2}{*}{ NT Central Australia } & \multirow{2}{*}{$\begin{array}{l}\begin{array}{l}\text { Far West } \\
\text { NSW }\end{array} \\
\text { ges } \pm \text { SE } \\
\text { alth centres) }\end{array}$} & \multirow[t]{2}{*}{ WA } & \multirow[t]{2}{*}{ North QLD } & \multirow[t]{2}{*}{ Total } \\
\hline & & & & & & \\
\hline Total No. of centres | adults & $24 \mid 708$ & $9 \mid 267$ & $5 \mid 150$ & $7 \mid 210$ & $17 \mid 504$ & $62 \mid 1839$ \\
\hline Total number of adults with recorded BP readings & 507 & 201 & 83 & 137 & 379 & 1307 \\
\hline $\begin{array}{l}\text { Percentage of adults with BP } \\
\text { readings } \geq 140 / 90 \mathrm{mmHg}\end{array}$ & $\begin{array}{l}9 \% \pm 2 \% \\
(0 \%-28 \%)\end{array}$ & $\begin{array}{l}8 \% \pm 2 \% \\
(4 \%-24 \%)\end{array}$ & $\begin{array}{l}24 \% \pm 7 \% \\
(0 \%-43 \%)\end{array}$ & $\begin{array}{l}9 \% \pm 2 \% \\
(0 \%-16 \%)\end{array}$ & $\begin{array}{l}27 \% \pm 6 \% \\
(7 \%-100 \%)\end{array}$ & $\begin{array}{l}15 \% \pm 2 \% * \\
(0 \%-100 \%)\end{array}$ \\
\hline $\begin{array}{l}\text { Percentage of adults with an } \\
\text { abnormal BP who had a repeated } \\
\text { BP check and/or subsequent } \\
\text { management plan }\end{array}$ & $\begin{array}{l}9 \% \pm 5 \% \\
(0 \%-100 \%)\end{array}$ & $\begin{array}{l}13 \% \pm 11 \% \\
(0 \%-100 \%)\end{array}$ & $\begin{array}{l}29 \% \pm 17 \% \\
(0 \%-67 \%)\end{array}$ & $\begin{array}{l}21 \% \pm 16 \% \\
(0 \%-100 \%)\end{array}$ & $\begin{array}{l}32 \% \pm 6 \% \\
(0 \%-100 \%)\end{array}$ & $\begin{array}{l}19 \% \pm 4 \% * \\
(0 \%-100 \%)\end{array}$ \\
\hline Total number of adults with recorded urine dipstick tests & 303 & 123 & 19 & 72 & 212 & 729 \\
\hline $\begin{array}{l}\text { Percentage of adults having } \\
\text { positive protein in urine tests }\end{array}$ & $\begin{array}{l}16 \% \pm 3 \% \\
(0 \%-50 \%)\end{array}$ & $\begin{array}{l}17 \% \pm 6 \% \\
(0 \%-60 \%)\end{array}$ & $\begin{array}{l}17 \% \pm 9 \% \\
(0 \%-50 \%)\end{array}$ & $\begin{array}{c}17 \% \pm 7 \% \\
(0 \%-50 \%)\end{array}$ & $\begin{array}{l}30 \% \pm 6 \% \\
(0 \%-92 \%)\end{array}$ & $\begin{array}{c}20 \% \pm 2 \% * \\
(0 \%-92 \%)\end{array}$ \\
\hline $\begin{array}{l}\text { Percentage of adults with an } \\
\text { abnormal urine test who had a } \\
\text { subsequent ACR test and/or } \\
\text { management plan }\end{array}$ & $\begin{array}{l}20 \% \pm 8 \% \\
(0 \%-100 \%)\end{array}$ & $\begin{array}{l}38 \% \pm 18 \% \\
(0 \%-100 \%)\end{array}$ & $\begin{array}{c}0 \% \\
(-)\end{array}$ & $\begin{array}{c}0 \% \\
(-)\end{array}$ & $\begin{array}{l}37 \% \pm 8 \% \\
(0 \%-100 \%)\end{array}$ & $\begin{array}{l}25 \% \pm 5 \% \\
(0 \%-100 \%)\end{array}$ \\
\hline Total number of adults with recorded blood glucose readings & 396 & 150 & 53 & 111 & 319 & 1029 \\
\hline $\begin{array}{l}\text { Percentage of adults with glucose } \\
\text { readings } \geq 5.5 \mathrm{mmol} / \mathrm{L}\end{array}$ & $\begin{array}{c}33 \% \pm 5 \% \\
(0 \%-89 \%)\end{array}$ & $\begin{array}{l}35 \% \pm 8 \% \\
(10 \%-67 \%)\end{array}$ & $\begin{array}{c}23 \% \pm 12 \% \\
(0 \%-67 \%)\end{array}$ & $\begin{array}{c}19 \% \pm 5 \% \\
(0 \%-35 \%)\end{array}$ & $\begin{array}{l}53 \% \pm 5 \% \\
(10 \%-94 \%)\end{array}$ & $\begin{array}{c}37 \% \pm 3 \% * \\
(0 \%-94 \%)\end{array}$ \\
\hline $\begin{array}{l}\text { Percentage of adults with an } \\
\text { abnormal glucose tests who had a } \\
\text { repeated glucose test and/or } \\
\text { subsequent management plan }\end{array}$ & $\begin{array}{c}22 \% \pm 6 \% \\
(0 \%-88 \%)\end{array}$ & $\begin{array}{c}11 \% \pm 8 \% \\
(0 \%-75 \%)\end{array}$ & $\begin{array}{c}19 \% \pm 10 \% \\
(0 \%-33 \%)\end{array}$ & $\begin{array}{c}14 \% \pm 9 \% \\
(0 \%-50 \%)\end{array}$ & $\begin{array}{l}23 \% \pm 6 \% \\
(0 \%-100 \%)\end{array}$ & $\begin{array}{l}19 \% \pm 3 \% \\
(0 \%-100 \%)\end{array}$ \\
\hline
\end{tabular}

$* P<0.05$ for comparison between regions.

** Further analyses showed no statistically significant differences in measures between Indigenous and non-Indigenous adults in Far West NSW.

Percentages are based on standard stratified samples from each health centre with equal numbers of males and females in three age bands and are not weighted for population size. 


\begin{tabular}{|c|c|c|}
\hline \multirow[b]{2}{*}{ Outcome measure } & \multicolumn{2}{|c|}{$\begin{array}{l}\text { Percent of total variation in outcome } \\
\text { measures attributable to: }\end{array}$} \\
\hline & $\begin{array}{c}\text { Health centre } \\
\text { level } \\
\text { characteristics }\end{array}$ & $\begin{array}{c}\text { Individual client level } \\
\text { characteristics }\end{array}$ \\
\hline \multicolumn{3}{|l|}{ Adherence to delivery of preventive services } \\
\hline \multicolumn{3}{|l|}{ Basic measurements done } \\
\hline Weight & $29 \%$ & $71 \%$ \\
\hline Height & $30 \%$ & $70 \%$ \\
\hline BMl & $47 \%$ & $53 \%$ \\
\hline Waist circumference & $42 \%$ & $58 \%$ \\
\hline Blood pressure & $22 \%$ & $78 \%$ \\
\hline \multicolumn{3}{|l|}{ Laboratory investigations done } \\
\hline Urinalysis & $24 \%$ & $76 \%$ \\
\hline Blood glucose level & $21 \%$ & $79 \%$ \\
\hline Pap smear (women only) & $20 \%$ & $80 \%$ \\
\hline STI: PCR for gonorrhoea \& chlamydia & $39 \%$ & $61 \%$ \\
\hline STI: Syphilis serology & $43 \%$ & $57 \%$ \\
\hline Oral health check done & $44 \%$ & $56 \%$ \\
\hline \multicolumn{3}{|l|}{ Brief interventions/advice done } \\
\hline Smoking & $28 \%$ & $72 \%$ \\
\hline Nutrition & $35 \%$ & $65 \%$ \\
\hline Alcohol & $30 \%$ & $70 \%$ \\
\hline Physical activity & $42 \%$ & $58 \%$ \\
\hline Mood (emotional well-being) & $34 \%$ & $66 \%$ \\
\hline Overall adherence & $31 \%$ & $69 \%$ \\
\hline \multicolumn{3}{|l|}{ Follow up of abnormal findings } \\
\hline $\begin{array}{l}\text { Proportion of adults with abnormal BP who had repeated BP check or documented plan to } \\
\text { repeat BP }\end{array}$ & $13 \%$ & $87 \%$ \\
\hline $\begin{array}{l}\text { Proportion of adults with abnormal urine tests who had ACR tests or documented plan to test } \\
\text { ACR }\end{array}$ & $33 \%$ & $67 \%$ \\
\hline $\begin{array}{l}\text { Proportion of adults with abnormal glucose tests who had repeated glucose tests or } \\
\text { documented plan to repeat }\end{array}$ & $38 \%$ & $62 \%$ \\
\hline
\end{tabular}

\section{Results}

Of 62 participating health centres, $47 \%$ were managed by a local or regional Aboriginal committee (board), with the remainder government funded/operated (Table $1)$. Sixty nine percent of centres did not have formal general practice accreditation and most (60\%) served populations of less than 1000 people.

Records of 1839 well adults were audited (Table 1). The mean age of these adults was 32 years and $49 \%$ were men. Around $90 \%$ or more of records from the NT, WA and North QLD centres were for Indigenous people compared to $43 \%$ from Far West NSW centres. Twenty eight percent of adults were documented as smokers and $23 \%$ had documented alcohol misuse. Ninety two percent of participants had a record of health centre attendance within the previous 24 months, with acute care the main reason for attendance and nurses as predominant health providers.
Overall delivery of scheduled services was 34\% (Table 2 ), with substantial variation in this measure between health centres (range 5-74\%) and moderate variation between regions (range 19-42\%).

For specific preventive services, variation in delivery was evident across different categories of services, different regions, and different health centres (Table 2). Overall, adherence was relatively high for weight and blood pressure measurement and blood glucose testing (50-70\%), followed by height measurement, urinalysis, pap smear and STI screening (30-40\%), and waist circumference measurement and brief intervention/counselling on lifestyle modification (20-30\%). Less attention was paid to oral health checks and brief intervention or counselling regarding emotional well being (15-18\%). However, the range between health centres for delivery of almost all of these services was from 0 - > $80 \%$. 
Table 6 Associations of health centre level and individual level factors with adherence to delivery of scheduled preventive services

\begin{tabular}{|c|c|c|c|}
\hline \multirow[b]{2}{*}{$\begin{array}{l}\text { Characteristic } \\
\text { (independent variables) }\end{array}$} & \multirow[b]{2}{*}{ Adherence to delivery (\%) } & \multicolumn{2}{|c|}{ Dependent variable } \\
\hline & & Unadjusted Coefficients (95\% Cl) & Adjusted Coefficients* $(95 \% \mathrm{Cl})$ \\
\hline \multicolumn{4}{|l|}{ Regions } \\
\hline NT Top End & $34 \%$ & Referent & Referent \\
\hline NT Central Australia & $42 \%$ & $8 \%(-6 \%, 23 \%)$ & $7 \%(-5 \%, 19 \%)$ \\
\hline Far West NSW & $19 \%$ & $-15 \%(-26 \%,-4 \%)$ & $-14 \%(-27 \%,-2 \%)$ \\
\hline WA & $28 \%$ & $-6 \%(-19 \%, 7 \%)$ & $3 \%(-12 \%, 18 \%)$ \\
\hline North QLD & $37 \%$ & $3 \%(-7 \%, 13 \%)$ & $9 \%(-1 \%, 18 \%)$ \\
\hline \multicolumn{4}{|l|}{ Health centre level characteristics } \\
\hline \multicolumn{4}{|l|}{ Locations } \\
\hline City $(n=150)$ & $23 \%$ & Referent & Referent \\
\hline Regional town $(n=360)$ & $25 \%$ & $2 \%(-7 \%, 12 \%)$ & $9 \%(-6 \%, 24 \%)$ \\
\hline Remote community $(\mathrm{n}=1329)$ & $38 \%$ & $15 \%(7 \%, 22 \%)$ & $18 \%(3 \%, 33 \%)$ \\
\hline \multicolumn{4}{|l|}{ Health service governance } \\
\hline Government $(n=980)$ & $33 \%$ & Referent & Referent \\
\hline Indigenous committee or board $(n=859)$ & $35 \%$ & $2 \%(-7 \%, 11 \%)$ & $13 \%(3 \%, 24 \%)$ \\
\hline \multicolumn{4}{|l|}{ General practice accreditation status } \\
\hline Not accredited $(n=1263)$ & $33 \%$ & Referent & Referent \\
\hline Currently accredited $(n=576)$ & $36 \%$ & $3 \%(-5 \%, 12 \%)$ & $6 \%(-1 \%, 13 \%)$ \\
\hline \multicolumn{4}{|l|}{ Sizes of populations served } \\
\hline$\leq 500(n=562)$ & $42 \%$ & Referent & Referent \\
\hline $501-999(n=507)$ & $34 \%$ & $-8 \%(-20 \%, 4 \%)$ & $-5 \%(-15 \%, 5 \%)$ \\
\hline$\geq 1000(n=770)$ & $28 \%$ & $-14 \%(-24 \%,-4 \%)$ & $-10 \%(-19 \%,-1 \%)$ \\
\hline \multicolumn{4}{|l|}{ Individual level characteristics } \\
\hline \multicolumn{4}{|l|}{ Age (years) } \\
\hline $15-24(n=627)$ & $32 \%$ & Referent & Referent \\
\hline $25-39(n=660)$ & $37 \%$ & $5 \%(2 \%, 9 \%)$ & $6 \%(2 \%, 9 \%)$ \\
\hline $40-54(n=552)$ & $33 \%$ & $1 \%(-2 \%, 4 \%)$ & $3 \%(-1 \%, 6 \%)$ \\
\hline \multicolumn{4}{|l|}{ Sex } \\
\hline Males $(n=909)$ & $31 \%$ & Referent & Referent \\
\hline Females $(n=930)$ & $37 \%$ & $6 \%(3 \%, 9 \%)$ & $6 \%(3 \%, 8 \%)$ \\
\hline $\mathrm{R}^{2}$ of the model ${ }^{* *}$ & & \multicolumn{2}{|c|}{$13 \%$} \\
\hline
\end{tabular}

Analyses of preventive service delivery between Indigenous and non-Indigenous adults in Far West NSW health centres showed no statistical difference in overall service delivery between the two groups. However, Indigenous adults were more likely to receive services related to BMI and waist circumference measurements, blood glucose testing and emotional wellbeing counselling.

On average, health centre-level documentation of an abnormal blood pressure reading ( $\geq 140 / 90 \mathrm{mmHg}$ ) was found in $15 \%$ of adults, proteinuria in $20 \%$, and abnormal blood glucose $(\geq 5.5 \mathrm{mmol} / \mathrm{L})$ in $37 \%$ (Table 4$)$. However, the range between health centres for these measures were $0-100 \%, 0-92 \%$ and $0-94 \%$ respectively. North Queensland health centres had higher rates of abnormal blood pressure, proteinuria and abnormal blood glucose compared with other regions $(\mathrm{P}<0.05$ for comparison with NT Top End). Of those with identified abnormal clinical findings, overall about $20-30 \%$ had a documented follow up check/test or management plan, but the range between services was $0-100 \%$.

Client level characteristics accounted for a large proportion of the variation in delivery of services and in follow up of abnormal findings: $69 \%$ for overall adherence to delivery of scheduled services (with a range of 53$79 \%$ for specific services); and between $62-87 \%$ for follow up of abnormal findings (Table 5).

Age and sex were both independently associated with overall delivery of services, with higher rates of delivery in the 25-39 year age group and in women (Table 6). 
Table 7 Associations of health centre level and individual level factors with follow-up of abnormal findings

\begin{tabular}{|c|c|c|c|c|c|c|c|c|c|}
\hline \multirow[b]{2}{*}{$\begin{array}{l}\text { Characteristic } \\
\text { (independent variables) }\end{array}$} & \multicolumn{9}{|c|}{ Dependent variables } \\
\hline & $\begin{array}{c}\text { Follow-up of } \\
\text { abnormal BP } \\
(\%)\end{array}$ & $\begin{array}{c}\text { Unadjusted } \\
\text { Risk Ratios } \\
(95 \% \mathrm{Cl})\end{array}$ & $\begin{array}{c}\text { Adjusted } \\
\text { Risk Ratios* } \\
(95 \% \mathrm{Cl})\end{array}$ & $\begin{array}{c}\text { Follow-up of } \\
\text { abnormal urine tests } \\
(\%)\end{array}$ & $\begin{array}{c}\text { Unadjusted } \\
\text { Risk Ratios } \\
(95 \% \mathrm{Cl})\end{array}$ & $\begin{array}{c}\text { Adjusted } \\
\text { Risk Ratios* } \\
(95 \% \mathrm{Cl})\end{array}$ & $\begin{array}{l}\text { Follow-up of abnormal } \\
\text { glucose tests }(\%)\end{array}$ & $\begin{array}{c}\text { Unadjusted } \\
\text { Risk Ratios } \\
(95 \% \mathrm{Cl})\end{array}$ & $\begin{array}{c}\text { Adjusted } \\
\text { Risk Ratios* } \\
(95 \% \mathrm{Cl})\end{array}$ \\
\hline \multicolumn{10}{|l|}{ Regions } \\
\hline NT Top End & $13 \%$ & Referent & Referent & $28 \%$ & Referent & Referent & $29 \%$ & Referent & Referent \\
\hline NT Central Australia & $13 \%$ & $0.9(0.2,3.2)$ & $1.5(0.3,4.6)$ & $40 \%$ & $1.4(0.5,2.7)$ & $2.3(0.9,3.2)$ & $18 \%$ & $0.6(0.1,2.3)$ & $0.6(0.1,2.2)$ \\
\hline Far West NSW & $28 \%$ & $2.1(0.4,5.4)$ & $0.9(0.2,3.6)$ & $0 \%$ & - & - & $13 \%$ & $0.5(0.1,1.7)$ & $0.5(0.1,1.5)$ \\
\hline WA & $15 \%$ & $1.2(0.2,3.9)$ & $1.2(0.1,5.1)$ & $0 \%$ & - & - & $13 \%$ & $0.5(0.1,1.6)$ & $0.6(0.2,1.5)$ \\
\hline North QLD & $26 \%$ & $2.0(0.7,4.3)$ & $4.1(1.6,6.3)$ & $42 \%$ & $1.5(0.8,2.3)$ & $1.9(0.8,2.9)$ & $18 \%$ & $0.6(0.2,1.5)$ & $0.8(0.2,1.9)$ \\
\hline \multicolumn{10}{|l|}{$\begin{array}{l}\text { Health centre level } \\
\text { characteristics }\end{array}$} \\
\hline \multicolumn{10}{|l|}{ Locations } \\
\hline City & $0 \%$ & - & - & $17 \%$ & Referent & Referent & $20 \%$ & Referent & Referent \\
\hline Regional town & $25 \%$ & Referent & Referent & $39 \%$ & $2.3(0.4,5.1)$ & $4.7(0.5,5.8)$ & $15 \%$ & $0.8(0.2,1.9)$ & $0.7(0.2,1.9)$ \\
\hline Remote community & $22 \%$ & $0.9(0.4,1.7)$ & $0.6(0.1,2.2)$ & $33 \%$ & $2.0(0.3,4.7)$ & $2.3(0.1,5.6)$ & $22 \%$ & $1.1(0.4,2.3)$ & $0.9(0.2,2.4)$ \\
\hline \multicolumn{10}{|l|}{ Health service governance } \\
\hline $\begin{array}{l}\text { Government funded/ } \\
\text { operated }\end{array}$ & $22 \%$ & Referent & Referent & $38 \%$ & Referent & Referent & $18 \%$ & Referent & Referent \\
\hline $\begin{array}{l}\text { Managed by local or } \\
\text { regional Indigenous } \\
\text { committee or board }\end{array}$ & $21 \%$ & $0.9(0.5,1.8)$ & $2.5(0.8,4.0)$ & $22 \%$ & $0.6(0.2,1.2)$ & $1.1(0.4,1.9)$ & $27 \%$ & $1.5(0.5,3.2)$ & $1.5(0.4,3.8)$ \\
\hline \multicolumn{10}{|l|}{$\begin{array}{l}\text { General practice } \\
\text { accreditation status }\end{array}$} \\
\hline Not accredited & $25 \%$ & Referent & Referent & $37 \%$ & Referent & Referent & $23 \%$ & Referent & Referent \\
\hline Currently accredited & $15 \%$ & $0.6(0.3,1.1)$ & $0.5(0.2,1.1)$ & $25 \%$ & $0.7(0.3,1.3)$ & $0.4(0.1,1.1)$ & $16 \%$ & $0.7(0.3,1.4)$ & $0.8(0.3,1.5)$ \\
\hline \multicolumn{10}{|l|}{ Sizes of populations served } \\
\hline$\leq 500$ & $31 \%$ & Referent & Referent & $28 \%$ & Referent & Referent & $30 \%$ & Referent & Referent \\
\hline $501-999$ & $19 \%$ & $0.6(0.3,1.3)$ & $0.5(0.2,1.1)$ & $34 \%$ & $1.2(0.6,2.1)$ & $1.0(0.5,1.8)$ & $19 \%$ & $0.7(0.2,1.7)$ & $0.7(0.3,1.6)$ \\
\hline$\geq 1000$ & $18 \%$ & $0.6(0.3,1.1)$ & $0.8(0.3,1.5)$ & $36 \%$ & $1.3(0.6,2.2)$ & $2.2(0.9,3.0)$ & $15 \%$ & $0.5(0.2,1.3)$ & $0.6(0.2,1.3)$ \\
\hline \multicolumn{10}{|l|}{$\begin{array}{l}\text { Individual level } \\
\text { characteristics }\end{array}$} \\
\hline \multicolumn{10}{|l|}{ Age (years) } \\
\hline $15-24$ & $17 \%$ & Referent & Referent & $36 \%$ & Referent & Referent & $15 \%$ & Referent & Referent \\
\hline 25-39 & $19 \%$ & $1.1(0.4,2.5)$ & $1.2(0.4,2.7)$ & $31 \%$ & $0.9(0.5,1.4)$ & $0.9(0.5,1.4)$ & $25 \%$ & $1.7(0.9,2.7)$ & $1.8(0.9,2.9)$ \\
\hline 40-54 & $25 \%$ & $1.5(0.6,2.8)$ & $1.6(0.7,3.1)$ & $32 \%$ & $0.9(0.4,1.5)$ & $0.9(0.3,1.7)$ & $22 \%$ & $1.5(0.9,2.3)$ & $1.7(0.9,2.8)$ \\
\hline \multicolumn{10}{|l|}{ Sex } \\
\hline Males & $22 \%$ & Referent & Referent & $34 \%$ & Referent & Referent & $20 \%$ & Referent & Referent \\
\hline Females & $21 \%$ & $1.0(0.6,1.5)$ & $0.9(0.5,1.4)$ & $33 \%$ & $1.0(0.6,1.5)$ & $0.9(0.5,1.5)$ & $22 \%$ & $1.1(0.7,1.6)$ & $1.1(0.8,1.6)$ \\
\hline
\end{tabular}

* Calculated using two-level random effects logistic regression models, with adjustment of other variables in the table. Odd Ratios generated from the models were converted into risk ratios using a published formula.[14]

Risk ratios significant at 0.05 level are shown in bold. 
Health centre level factors which were independently associated with higher level of delivery of services were location (remote community vs city), community population size ( $\leq 500$ vs $\geq 1000$ ), region (Top End vs FW NSW) and governance (Indigenous committee/board operated vs government).

For follow-up of abnormal findings, North Queensland had higher rates of follow-up of abnormal BP (Table 7) [14]. No other health centre or individual characteristics showed significant associations with follow-up of abnormal results.

\section{Discussion}

There is substantial room to improve the quality of preventive care to Indigenous adults in many locations across Australia - in terms of overall delivery of services, in delivery of a range of specific services and in follow up of abnormal findings from routine health checks. Overall, it appears that about one third of the recommended preventive services were delivered to clients in participating health centres. Variation in overall delivery of guideline scheduled services between health centres is striking, with the lowest adherence to delivery being 5\% and the highest being $74 \%$. For specific important measures such as BP screening, overall $71 \%$ of adults have a record within the previous two years. However the variation between centres of $23-100 \%$ reveals a critical requirement for action in some health centres. The generally small proportion of clients with records of or plans for follow-up of abnormal clinical findings among these 'well' adults also highlights an important area for improvement.

Limitations of this study include: 1) Health centres were not randomly selected and their participation in the project was on a voluntary basis and enrolment was staggered over a period of some years. Therefore, these data are not representative for the regions involved and differences between health centres may be partly the result of introduction of new policies over time. A longitudinal analysis including services with more than three years of data through participating in this project will be reported separately. 2) We relied on clinical medical records to retrieve preventive care data, which may underestimate actual service delivery if delivered services are not recorded in clinical records. While failure to document services may mean that services are delivered at higher levels than reflected in our data, the failure to document delivered services is itself a significant barrier to continuity and coordination of care and in preventing duplication and over servicing - especially in areas of high workforce turnover. Failure to document delivered services is therefore in itself a deficiency in quality of care. 3) The unweighted age and sex stratified random samples are designed to facilitate analysis of quality of care between communities. Estimates based on this sampling approach may differ from sampling approaches designed to provide population estimates.

The pattern of delivery of different services (with blood pressure checks and blood glucose testing for well adults being relatively high, followed by delivery of urinalysis, pap smear and STI screening, provision of brief interventions/counselling related to lifestyle change, and with lowest levels of delivery for oral health checks and counselling on emotional well being) to some extent reflects a gradient in strength of evidence for the preventive services specified in best practice guidelines $[2,15]$. Practitioners appear less likely to provide some services where the availability of referral services (e.g. for dental and mental health care [16]) is limited. However, the low proportion of adults identified as smokers in relation to known smoking rates in these communities is an example of an important gap in documentation of major risk factors where there are relatively simple primary care interventions with a reasonably well established evidence base.

The comparability of these findings with similar studies $[17,18]$ is limited by the inclusion in these studies of the general adult population, while our study focuses on an age and sex stratified (unweighted) random samples of well adults. People with chronic illness are likely to have increased contact with the health systems and more opportunities for receiving preventive services, so delivery of preventive services to well adults might be expected to be lower than for people with chronic illness. As indicated above, the stratified random sample used in our study may also not be representative of the study populations in each community or of the study populations for all communities combined. Bearing these restrictions on comparability with other studies in mind, we note that delivery of some services (such as $\mathrm{BP}$ ) in our study population compares reasonably well. However, the generally low levels of delivery of care, the well known burden of chronic disease in this population, the importance of early detection and treatment, and the high rates of attendance by our study population at primary care centres mean that many important opportunities are being missed and there is clearly a need for better delivery of preventive services.

High prevalence of health problems among "healthy adults" and low follow-up of identified problems are of significant concern. Similar to previous reports from Indigenous primary care settings $[7,8]$, about $20 \%-40 \%$ of the participating "healthy adults" in our study had abnormal blood pressure, abnormal blood sugar levels, or proteinuria. This highlights the importance and necessity of systematically implementing preventive care for adults in Indigenous communities for early detection and management of preventable chronic disease. A 
parallel priority in preventive care is to effectively follow up and manage the abnormal conditions identified. Failure to follow up and implement management plans means resources and efforts invested in regular checking and screening of well adults are wasted and cannot be translated into improved health outcomes.

Our analysis of variation in preventive care indicates health centre level and individual client level factors have a similar level of influence on delivery of preventive care. The finding that accreditation of services is not clearly associated with quality of care is consistent with other research on this topic $[19,20]$, and indicates the need for a more active approach to quality improvement (e.g. routine use of clinical data to monitor and improve quality of care; ongoing engagement of health centre staff in service planning, system redesign and implementation of improvement initiatives). The finding that delivery of preventive services is better in remote locations and worse in health centres with large service populations is likely to be at least partly due to greater use of a number of different providers by clients living in non-remote settings or larger centres. The finding that delivery of preventive services is better in community controlled services than government managed services supports the contention that community control (through its philosophy, organisation or funding) facilitates quality and access to care [21].

The substantial proportion of variation in preventive care attributable to client level factors points to the importance of health centre systems to deliver care in a way that most effectively meets the varying needs of individual clients. Regarding client level factors, we only collected demographic information of participants (i.e. age, sex and Indigenous status). Male participants appeared less likely to access preventive services than females in our study. This may reflect the perceptions of many Indigenous men who consider health centres as "women's places" [22], as health centres in remote communities are predominantly staffed by females. Gender appropriate workforce and infrastructure may encourage Indigenous men to better use of health services. Other client level factors, such as their health literacy [23], perceptions of physical, social and cultural accessibility of the centre, and factors which influence a client's relationship with health centre staff [24], are important influences on the delivery or uptake of preventive care. These questions need to be investigated in future research, as well as associations of specific health centre system factors $[6,25]$ with preventive care.

Beyond addressing potential health centre and client level factors, a supportive health policy has been recognised as critical to the implementation of preventive care to populations [26]. The introduction of a new Medicare item (item 710) in 2004 for health assessment of Indigenous people aged 15-54 years has been welcomed as an example of innovative policy in Indigenous health [27]. However, the impact of this measure is unclear and the longitudinal analysis of the $\mathrm{ABCDE}$ data should provide some evidence in this area. Previous research indicates that the Medicare rebates for providing preventive care may have less effect in motivating practitioners working in remote Indigenous community health centres who are usually in salaried positions [6]. More recent policy developments include new legislation which authorises practice nurses and Aboriginal Health Workers to access some Medicare items (e.g. for provision of immunisation and follow up services for Indigenous people after health assessment) [28], and introduction of the Indigenous Practice Incentives Program (PIP) to encourage population-based care [29]. Further refinement of health policies includes strengthening direct financial and workforce support to health centres based on needs of defined populations [30].

\section{Conclusions}

There is great potential to improve delivery of preventive services to well adults in Indigenous primary care settings. Particular attention should be given to improving follow-up of abnormal clinical findings identified by preventive health assessments. The national collaborative approach that underpins the data presented in this paper provides a significant opportunity to advance understanding of variation in care and to develop and examine the effect of innovative strategies to enhance the quality of care for Indigenous Australians.

\section{Acknowledgements}

This project would not be possible without the active support, enthusiasm and commitment of staff and management of the participating health services and the contribution made by the wider ABCD project team. The $A B C D$ project was supported by funding from the Cooperative Research Centre for Aboriginal Health and the Commission for Safety and Quality in Health Care. The work of a number of people with key roles in the project is supported by their employing organisations, including State and Territory governments and community controlled health organisations. DS's work is supported by a National Health and Medical Research Council (NHMRC) Capacity Building in Population Health Grant and NHMRC Postdoctoral Fellowship (\#569919). RB's work is supported by an NHMRC Research Fellowship.

\section{Author details}

'Menzies School of Health Research, Charles Darwin University, Darwin NT, Australia. ${ }^{2}$ School of Medicine, University of Queensland, Brisbane QLD, Australia. ${ }^{3}$ Northern Territory Department of Health and Families, Darwin NT, Australia. ${ }^{4}$ Queensland Department of Health, Cairns QLD, Australia. ${ }^{5}$ Maari Ma Health Aboriginal Corporation, Broken Hill NSW, Australia. ${ }^{6}$ Curtin University, Perth WA, Australia. ${ }^{7}$ Baker IDI Heart and Diabetes Institute (Alice Springs), Alice Springs NT, Australia. ${ }^{8}$ Western Australia Department of Health, Perth WA, Australia. ${ }^{9}$ Aboriginal Health Council of Western Australia, Perth WA, Australia.

\section{Authors' contributions}

$\mathrm{RB}$ played a lead role in conceptualisation of study design, development of measurement tools, project management, and revising of the manuscript. 
DS played a major role in reviewing the literature and conceptualisation, conducted data analysis, and drafted the manuscript. CC, AB, TW and ST and HB contributed to study design and facilitated engagement of health services. MD and LO contributed to study design and development of measurement tools. MD, LO, RK, CK, RC, HL, JH carried out field work and conducted data collection. All authors contributed to the interpretation of findings, read and approved the final manuscript.

\section{Competing interests}

The authors declare that they have no competing interests.

Received: 14 May 2010 Accepted: 1 June 2011 Published: 1 June 2011

\section{References}

1. Australian Institute of Health and Welfare: Chronic diseases and associated risk factors in Australia Canberra: AlHW; 2006.

2. National Aboriginal Community Controlled Health Organisation: National guide to a preventive health assessment in Aboriginal and Torres Strait Islander peoples Melbourne: Royal Australian College of General Practitioners; 2005

3. Australian Government Department of Health and Ageing: MBS Primary Care Items: Medicare Health Assessment for Aboriginal and Torres Strait Islander People - Fact Sheet [http://www.health.gov.au/internet/main/ publishing.nsf/Content/mbsprimarycare_ATSI_MBSitem715], Accessed on 13/09/2010.

4. Australian Government Preventative Health Taskforce: Australia: The Healthiest Country by 2020 - National Preventative Health Strategy - Overview Canberra: Australian Government Preventative Health Taskforce; 2009.

5. Bailie RS, Togni SJ, Si D, Robinson G, d'Abbs PH: Preventive medical care in remote Aboriginal communities in the Northern Territory: a follow-up study of the impact of clinical guidelines, computerised recall and reminder systems, and audit and feedback. BMC Health ServRes 2003, 3(1):15.

6. Si D, Bailie RS, Dowden M, O'Donoghue L, Connors C, Robinson GW, Cunningham J, Condon JR, Weeramanthri TS: Delivery of preventive health services to Indigenous adults: response to a systems-oriented primary care quality improvement intervention. Med J Aust 2007, 187(8):453-457.

7. Miller G, McDermott R, McCulloch B, Leonard D, Arabena K, Muller R: The Well Person's Health Check: a population screening program in indigenous communities in north Queensland. Aust Health Rev 2002, 25(6):136-147.

8. Spurling GK, Hayman NE, Cooney AL: Adult health checks for Indigenous Australians: the first year's experience from the Inala Indigenous Health Service. Med J Aust 2009, 190(10):562-564.

9. Kelaher M, Dunt D, Thomas D, Anderson I: Comparison of the uptake of health assessment items for Aboriginal and Torres Strait Islander people and other Australians: implications for policy. Aust New Zealand Health Policy 2005, 2:21.

10. Bailie R, Si D, Connors C, Weeramanthri T, Clark L, Dowden M, O'Donohue L, Condon J, Thompson S, Clelland N, Nagel T, Gardner K, Brown A: Study protocol: Audit and Best Practice for Chronic Disease Extension (ABCDE) Project. BMC Health Serv Res 2008, 8:184.

11. Central Australian Rural Practitioner Association: CARPA standard treatment manual: a clinic manual for primary health care practitioners in remote and rural communities in Central and Northern Australia Alice Springs: Central Australian Rural Practitioner Association; 2003.

12. StataCorp: Stata longitudinal/panel data reference mmanual. Release 10 College Station, TX: StataCorp LP; 2007.

13. Snijders $T A B, R$ J: Multilevel analysis: an introduction to basic and advanced multilevel modeling Thousand Oaks, CA: Sage; 1999

14. Zhang J, Yu KF: What's the Relative Risk?: A Method of Correcting the Odds Ratio in Cohort Studies of Common Outcomes. JAMA: The Journal of the American Medical Association 1998, 280(19):1690-1691.

15. The National Preventive and Community Medicine Committee of the Royal Australian College of General Practitioners: Guidelines for preventive activities in general practice. Aust Fam Physician 2002, 31:S1-S61.

16. Australian Bureau of Statistics, Australian Institute of Health and Welfare: The health and welfare of Australia's Aboriginal and Torres Strait Islander peoples, 2008 Canberra: Commonwealth of Australia; 2008.
17. McGlynn EA, Asch SM, Adams J, Keesey J, Hicks J, DeCristofaro A, Kerr EA: The quality of health care delivered to adults in the United States. NEnglJMed 2003, 348(26):2635-2645.

18. Schoen C, Osborn R, Huynh PT, Doty M, Davis K, Zapert K, Peugh J: Primary care and health system performance: adults' experiences in five countries. Health Aff (Millwood) 2004, , Suppl Web Exclusives: W4-487-503.

19. Greenfield D, Braithwaite J: Health sector accreditation research: a systematic review. Int J Qual Health Care 2008, 20(3):172-183.

20. Ovretveit J, Gustafson D: Using research to inform quality programmes. BMJ 2003, 326(7392):759-761.

21. National Aboriginal and Torres Strait Islander Health Council (NATSIHC): National Strategic Framework for Aboriginal and Torres Strait Islander Health: Framework for action by Governments Canberra: NATSIHC; 2003.

22. McCoy B: Holding Men: Kanyirninpa and the health of Aboriginal men Canberra: Aboriginal Studies Press; 2008.

23. Smylie J, Williams L, Cooper N: Culture-based literacy and Aboriginal health. Can J Public Health 2006, 97(Suppl 2):S21-25.

24. Foster $\mathrm{CH}$ : What nurses should know when working in Aboriginal communities. Can Nurse 2006, 102:28-31.

25. Glasgow RE, Orleans $C T$, Wagner EH: Does the chronic care model serve also as a template for improving prevention? Milbank Q 2001, 79(4):579-612, iv-v.

26. Couzos S, Murray R: Aboriginal primary health care: an evidence-based approach Melbourne: Oxford University Press; 2008

27. Mayers NR, Couzos S: Towards health equity through an adult health check for Aboriginal and Torres Strait Islander people: an important Australian initiative that sets an international precedent. Med J Aust 2004, 181(10):531-532.

28. Medicare Australia: Nurse practitioners and midwives [http://www. medicareaustralia.gov.au/provider/other-healthcare/nurse-midwives.jsp], accessed 14 September 2010.

29. Medicare Australia: Practice Incentives Program (PIP) - Indigenous Health Incentive [http://www.medicareaustralia.gov.au/provider/incentives/pip/files/ 2864-pip-indigenous-health-incentive-guidelines-5-5-11.pdf], Accessed 30 May 2011.

30. Australian Government Preventative Health Taskforce: Australia: The Healthiest Country by 2020 - National Preventative Health Strategy - the roadmap for action Canberra: Australian Government Preventative Health Taskforce; 2009.

\section{Pre-publication history}

The pre-publication history for this paper can be accessed here: http://www.biomedcentral.com/1472-6963/11/139/prepub

\section{doi:10.1186/1472-6963-11-139}

Cite this article as: Bailie et al:: Variation in quality of preventive care for well adults in Indigenous community health centres in Australia. BMC Health Services Research 2011 11:139.

\section{Submit your next manuscript to BioMed Central and take full advantage of:}

- Convenient online submission

- Thorough peer review

- No space constraints or color figure charges

- Immediate publication on acceptance

- Inclusion in PubMed, CAS, Scopus and Google Scholar

- Research which is freely available for redistribution

Submit your manuscript at www.biomedcentral.com/submit
C BioMed Central 